\title{
Results of the Treatment of Talar Neck Fractures in a West African Hospital
}

\author{
AD Kacou, MD Orth, EJB Sié, MD Orth, Y Lambin, MD Orth \\ Department of Orthopaedic Surgery, University of Yopougon Teaching Hospital, Abidjan, Côte d'Ivoire
}

\begin{abstract}
Talar neck fractures (TNFs) are complex injuries and treatment is fraught with complications. We retrospectively studied 18 patients with TNFs: 6 Hawkins type-I, 7 type-II, 4 type-III, and 1 type-IV. Five patients with type-I and 2 with type-II fractures were treated conservatively. The remaining 11 patients underwent operative treatment. Open reduction via posterolateral approach was performed in 10 patients while closed reduction was used in one patient. All operatively reduced fractures were stabilized with screws. The following postoperative complications were encountered: wound dehiscence $(\mathrm{n}=1)$, avascular necrosis $(\mathrm{n}=2)$, and post-traumatic osteoarthritis $(\mathrm{n}=12)$. Fifteen patients were available for final evaluation at a mean followup period of three years (range 10 months to 6 years). Using the American Orthopaedic Foot and Ankle Society (AOFAS) score, the mean for type-I fracture was 96.4 points, 80.2 points for type-II, 68.5 points for type-III and 55 points for type-IV fractures. TNFs remain a devastating injury as the prognosis and outcomes of treatment continued to be hampered by the consequences of injury.
\end{abstract}

Key Words:

Developing World, Talar Neck Fractures, Treatment Outcome

\section{INTRODUCTION}

Talar neck fractures (TNFs) are rare and predominantly result from high-energy trauma such as motor vehicle accidents and fall from a height. A non-displaced fracture can be treated conservatively while most displaced fractures require open reduction and internal fixation (ORIF) ${ }^{1-3}$. Studies conducted in developed countries indicate that treatment of TNFs is fraught with complications that compromise clinical outcomes, particularly malunion, avascular necrosis (AVN) and post-traumatic osteoarthritis $(\mathrm{OA})^{4-6}$. However, good-to-excellent results were reported in a series of 18 out of 22 patients evaluated at a mean followup period of 4.4 years ${ }^{7}$. This present study was undertaken to evaluate the outcomes of TNFs treated at a single institution with limited financial resources and operating facilities in the west African region ${ }^{8}$.

\section{MATERIALS AND METHODS}

\section{Patients}

This retrospective study included 18 patients treated for TNF between January 1996 and December 2009. There were 12 men and 6 women, aged between 16 and 56 years (mean 25 years). Mechanisms of injury were road traffic accident $(n=12)$, fall from a height $(n=5)$ and sports-related trauma $(n=1)$. Plains radiographs were used for the assessment of fractures and evaluation of treatment results. Neither computerized tomography scans (CT-scans) nor magnetic resonance imaging (MRI) was used. Fractures were classified according to modified Hawkins classification system $^{1,2}$. There were 6 minimally displaced fractures (typeI), 7 displaced fractures with subtalar subluxation (type-II), 4 fractures with tibiotalar subluxation (type-III) and 1 fracture with pan-talar subluxation (type-IV). Fourteen fractures were closed injuries, while open fractures of grade-I and -II were seen in four patients. Two patients had additional ipsilateral tibial and femoral shaft fractures, and one had contralateral Lisfranc injury. Associated fractures were treated with ORIF. Seven of 18 patients were seen primarily by our team within the 24 hours following the injury.

\section{Treatment}

Patients with closed fractures presented with a mean delay of 8 days (range 1 to 15 days) after the trauma. Most open fractures underwent operative procedures within 24 to 72 hours of the injury. The following treatment protocols were used for treatment of different fractures:

Type I - Treatment consisted of a short leg cast (SLC) with the foot in neutral position for seven weeks followed by foot and ankle rehabilitation after removal of cast (in five patients). In one patient, the fracture was treated by closed manipulative reduction (CMR) and percutaneous screw fixation using a posterolateral approach under image intensifier guidance. Ankle mobilisation was allowed two weeks after the operation. Weight-bearing ambulation was allowed at 12 weeks after the injury.

Type II - Two patients were treated by closed reduction and SLC similar to the protocol used for type-I fractures. In five patients ORIF (screw fixation) was performed using an anteromedial approach. A SLC was applied after removal of 
sutures; patients were kept non-weight bearing for 16 weeks ankle mobilisation.

Type III - All the patients $(\mathrm{n}=4)$ underwent operative setting of open fracture $(n=2)$, extensive wound debridement; fracture was accessed through the same wound. Patients with closed fracture underwent ORIF with screw fixation via an anteromedial approach $(\mathrm{n}=1)$ or a combined anteromedial and anterolateral surgical approach $(\mathrm{n}=1)$. The affected leg was immobilized in a SLC for 18 weeks before graduated full weight-bearing ambulation and ankle-foot mobilization was permitted.

Type IV - The patient had an open fracture treated surgically with ORIF and screw fixation after extensive debridement. The period of non-weight bearing ambulation was extended to 32 weeks.

\section{Evaluation}

Evaluation at follow-ups included identification of complications and radiographic assessment of union or complications of union. The radiographs were taken at 6,12 , 24, 36 and 48 months post-treatment. Subsequent radiographs for identification of $\mathrm{AVN}$ and post-traumatic $\mathrm{OA}$ were taken semi-annually until the last visit. AVN was defined on plain radiographs as an absence of Hawkins sign over the talar dome. Post-traumatic OA was defined as the presence of osteophytes, narrowing of joint space and subchondral sclerosis or cysts.

Overall functional outcome was assessed using the American Orthopaedic Foot and Ankle Society score (AOFAS) based on pain (40 points), function (50 points), and alignment (10 points $)^{9}$. An excellent result was assigned for a score of 90100 points, good for $75-89$ points, fair for $60-74$ points and poor for less than 60 points.

\section{RESULTS}

Three patients (two type-I and one type-II) were lost to followup at an average of 3 years (range, 10 months to 6 years). The outcomes of treatment for the remaining 15 patients are presented according to fracture type.

Type I - Fracture union was attained at an average of 10 weeks for all cases. The mean AOFS functional score of 96.4 points (range 90 to 98 ) indicated an excellent outcome for type-I fractures. However, two out of six patients showed radiographic evidences of post-traumatic OA of the subtalar joint.

Type II - Union was achieved at an average of twelve weeks and functional outcome was graded as good (mean AOFS score of 80.2). Mid-term radiographs showed post-traumatic OA of the subtalar joint in two patients, ankle in one and talonavicular joint in three patients.
Type III - Surgical site infection with wound dehiscence developed in one patient who had a combined approach for ORIF. This was successfully managed with oral antibiotics and wound care. Two patients demonstrated radiographic evidence of union twelve weeks after the injury and three patients had varus malunion. The AOFS functional score for this group was fair (mean 68.5 points). Mid- term radiographs revealed subtalar $(n=2)$ and ankle $(n=1)$ OA. Symptomatic ANV necrosis of the talus with severe pain was seen in one patient two years after the injury. The patient however, deferred ankle arthrodesis.

Type IV - The patient had a poor functional AOFS score of 55 points. Post-traumatic OA of the ankle and talonavicular joint was seen as AVN progressed.

\section{DISCUSSION}

Treatment of TNFs aimed at preventing the inevitable complications continues to challenge the orthopaedic surgeon ranging from appropriate diagnosis to the execution of surgical treatment. A true type-I fracture is probably rare and is difficult to differentiate from a type-II fracture on plain radiographs. The decision to embark on costly CTscans or MR imaging to accurately diagnose a type-I or even type-II injury is frequently considered to be unnecessary. A fracture with clear displacement of even 1 to $2 \mathrm{~mm}$ should be considered type-II rather than type-I fracture ${ }^{3}$. The majority of patients who develop post-traumatic OA had either a typeI or type-II fracture.

Prompt and anatomical reduction within 6 to 8 hours following the injury and stable osteosynthesis are the mainstays of treatment for displaced TNFs with the aims of achievement of primary bone healing and thus minimised complications ${ }^{10,11}$. A delay in treating these fractures was a striking point of the current study. The classic reason for postponing surgery for TNFs is concomitant soft tissue oedema. In the present study, patients presented at the hospital as soon as possible but the treatment was delayed for economic reasons as patients must pay out of pocket for all medical care due to the lack of a national health insurance program. In our hospital setting, logistically one theatre is dedicated to orthopaedics. This theatre was used for both trauma and elective surgeries with planned elective lists carried out in the morning and the remainder of the theatre time allocated for orthopaedic trauma surgery. In the study by Low et al. (1998), the operations were performed as early as possible with open fractures treated within 8 hours and closed fractures at an average of 13.8 hours after injury. However, Lindvall et al. (2004) in a series of 26 fractures postulated that the time to surgical fixation should be minimised, although a delay does not appear to adversely affect the outcome, especially with regard to the development of AVN. 
The limitations of our study include the small size of the sample, retrospective nature of the study and inability to utilize modern imaging modalities to appropriate detect complications following injury and surgery. We were aware that it is difficult to identify early changes of AVN on plain radiographs. Osteophytes and subchondral cysts are late signs of post-traumatic OA or AVN. Despite limitations, the present results are in agreement with the experience of other authors. Firstly in support of the studies by Hawkins and Canale and Kelly, an anteromedial approach is the most versatile approach for ORIF ${ }^{1,2}$. It seems that less satisfactory results occurs in patients with the most severe injury ${ }^{13,14}$. Thirdly, development of post-traumatic OA seems to occur regardless of fracture type. This consequence was seen in almost all patients as reported by Lindvall et al. (2004), and can probably be attributed to the initial articular damage sustained in severe fractures or dislocations as well as injuries to the neighbouring soft tissue ${ }^{15}$. This is also ascribed to prolonged immobilisation (range, 12 to 32 weeks) required for prevention of talar dome collapse secondary to AVN. In our community setting, cast immobilisation reduced the risk of subsequent manipulation of the injured limb by traditional healers ${ }^{16}$.

\section{CONCLUSION}

The current study reflects a snapshot of clinical practice in a typical hospital setting in a developing nation. The results of this series should be interpreted in the light of the aforementioned limitations, but are in overall agreement with previous reports that the poorest functional results occur in patients with the most severe injury type. A prospective study enrolling a large population size in our region of Africa with attention paid to the relationship between the types of fracture and the delay of treatment as well as functional outcome will provide meaningful insight into the appropriate management of talar neck fractures in developing countries. 


\section{REFERENCES}

1. Hawkins LG. Fractures of the neck of the talus. J Bone Joint Surg 1970; 52: 991-1002.

2. Canale ST, Kelly FB. Fractures of the neck of the talus. Long-term evaluation of seventy-one cases. J Bone Joint Surg 1978; 60: 143-56.

3. Fortin PT, Balazsy JE. Talar fractures: Evaluation and treatment. J Am Acad Orthop 2001; 9: 114-27.

4. Rammelt S, Zwipp H. Talar neck and body fractures. Injury 2009; 40: 120-35.

5. Sanders DW, Busam M, Hattwick E, Edwards JR, McAndrew MP, Johnson KD. Functional outcomes following displaced talar neck fractures. J Orthop Trauma 2004; 18: 265-70.

6. Vallier HA, Nork SE, Barei DP, Benirschke SK, Sangeorzan BJ. Talar neck fractures: Results and outcomes. J Bone Joint Surg 2004; 86: 1616-24.

7. Low CK, Chong CK, Wong HP, Low YP. Operative treatment of displaced talar neck fractures. Ann Acad Med Singapore 1998; 27: 763-6

8. Dormans JP, Fischer RC, Pill SG. Orthopaedics in the developing world: Present and future concerns. J Am Acad Ortho Surg 2001; 9: 289-96.

9. Kitaoka HB, Alexander IJ, Adelaar RS, Nunley JA, Myerson MS, Sanders M. Clinical rating system for the ankle,hindfoot, midfoot, hallux and lesser toes. Foot Ankle Int 1994; 15: 349-53.

10. Grob D, Simpson LA, Weber BG, Bray T. Operative treatment of displaced talar neck fractures. Clin Orthop 1985; 199: 88-96.

11. Comfort TH, Behrans F, Gaither DW, Denis F, Sigmond M. Long-term results of displaced talar neck fractures. Clin Orthop 1985; 199: 81-7.

12. Lindvall E, Haidukewych F, DiPasquale T, Herscovici D, Sanders R. Open reduction and stable fixation of isolated, displaced talar neck and body fractures. J Bone Joint Surg 2004; 86-A: 2229-34.

13. Garcia-Rey E, Sanz-Hospital FJ, Galdran FJ, Cano-Egea JM, Alcazar LFL. Talar neck fractures: Results and complication by type. J Foot Ankle Surg 2002; 8: 203-8.

14. Schulze W, Richter J, Russe O, Ingelfinger P, Muhr G. Surgical treatment of talus fractures: A retrospective study of 80 cases followed for 1-15 years. Acta Orthop Scand 2002; 73: 344-51.

15. Saudan M, Peter R, Delmi M. Talar neck fractures. A 9-year follow-up of 40 cases. Rev Chir Orthop Reparatrice Appar Mot 2002; 88: 168-76.

16. Onuminya JE. The role of the traditional bonesetter in primary fracture care in Nigeria. S Afr Med J 2004; 94: 652-8. 